\title{
Leader-Follower Formations: Uncalibrated Vision-Based Localization and Control
}

\author{
Gian Luca Mariottini, Fabio Morbidi, Domenico Prattichizzo \\ Dipartimento di Ingegneria dell' Informazione \\ Università di Siena \\ Via Roma 56, 53100 Siena, Italy \\ Email: $\{$ gmariottini,morbidi,prattichizzo $\} @$ dii.unisi.it
}

\author{
George J. Pappas, Kostas Daniilidis \\ GRASP Laboratory, \\ University of Pennsylvania \\ Philadelphia, PA 19104, USA \\ Email: \{kostas,pappasg\}@grasp.upenn.edu
}

\begin{abstract}
This paper focuses on leader-follower formations of mobile robots equipped with panoramic cameras and extend earlier works in the literature addressing both the visionbased localization and control problems. First, a new sufficient analytical condition for localizability is proved and used to shed light on the geometrical meaning of formation localization using uncalibrated vision sensors, here performed with the Unscented Kalman Filter. Second, we design a feedback control law based on dynamic extension in order to extend the applicability of our control scheme also to the case of distant robots.
\end{abstract}

\section{INTRODUCTION}

Distributed vision systems or camera networks mounted on robotic platforms are ubiquitous today in many applications including mapping of unknown environments, distributed manipulation, exploration and autonomous navigation. In the last few years, we witnessed an increasing interest in formation control topics. With formation control, we simply mean the problem of controlling the relative position and orientation of camera-robots in a group while allowing the group to move as a whole [2]. In this sense, formation control needs the solution of the localization problem, i.e., the estimation of the relative position and orientation of all robots with respect to a reference coordinate system. An inexpensive and challenging way to solve this problem is to use entirely passive vision systems -off-theshelf cameras- which can provide only the projection of the scene points. This is dual to wireless networks where only distance or some function of distance (inverse square) can be measured [3], [8].

The localization problem with vision sensors is intrinsically nonlinear [1], in fact linearized approximations can be non-observable, while tools from differential nonlinear systems theory prove the possibility to reconstruct the state. This localization problem is often referred to as the observability of perspective dynamical systems [10], [11] and can be embedded in the more general problem of current state estimation using input-output measurements. In [4], the state estimation for a single robot is approached using a Luenberger-like nonlinear observer, based on the projection of stationary landmarks in the environment. In [16] the localization problem for a team of nonholonomic mobile robots with calibrated vision sensors, is addressed using motion segmentation techniques based on optical-flow.
Das et al. [5] have recently presented an interesting centralized framework for vision-based formation control where the leader moves along predefined trajectories, while the followers are to maintain a desired relative posture (distance and angle) with respect to the leader. However, an underlying assumption consists in assuming that the leader-follower distance can be provided directly by the calibrated panoramic camera mounted on the robots.

In [14], we relaxed the above assumption and exploited the Extended Output Jacobian (EOJ) to analytically prove a sufficient condition on the formation localizability with uncalibrated panoramic cameras, providing only the viewangle between each robot pair (and not the distance).

In this paper, we address both the localization and the control problems for a leader-follower formation of nonholonomic mobile robots equipped with panoramic cameras. In particular, we list here the innovative contributions of the present work:

- Differently from [5] and [14], we assume that the only distinguishable feature detected by each camera is the view-angle to the other observed robot's center of gravity (or centroid). This is a convenient choice (particularly suited, e.g., for airplane formations) because the centroid can be easily computed with standard computer vision techniques [7] also in the case of distant robots. In this case, the EOJ theory allows us to prove the feasibility of the formation localization and gives a very intuitive geometrical interpretation of this property.

- Differently from [14], the localization has been approached here using the Unscented Kalman Filter (UKF) [13], a derivative-free alternative to the extended Kalman filter (EKF). UKF is particularly suitable for multirobot localization since its equations do not change as new robots join the formation. Moreover, system nonlinearities are approximated with higher accuracy than the EKF.

- The state-tracking control law proposed in [5] does not apply when observing robot centroids. To overcome this drawback, we designed a feedback control law based on dynamic extension, whose effectiveness has been proved through simulation experiments with noisy data. 




Fig. 1. The communication network and the information flow between the leader and the followers.

We emphasize that, due to the aims of this work and space constraints, we are not interested in presenting experimental results which are expected to be comparable to those in [5], due to the similarity of the leader-follower setup.

The paper is organized as follows. In Sect. II the general leader-follower kinematic model and some basic assumptions are introduced. In Sect. III we present the visionbased observability study for leader-follower formations. In Sect. IV we introduce the Unscented Kalman Filter. In Sect. V we design the feedback control via dynamic extension. Simulation results are discussed in Sect. VI. In Sect. VII the major contributions of the paper are summarized and future research lines are highlighted.

Distributed architecture/communication network:

We assume that both the state estimation process and the control law computation are centralized on the leader $\langle L\rangle$ which transmits to the $i$ th follower $\left\langle F_{i}\right\rangle$ the velocity control $\left[v_{F i} \omega_{F i}\right]^{T}$ needed to maintain the formation (see Fig. 1). The view-angle $\eta_{i}$ from $\left\langle F_{i}\right\rangle$ to $\langle L\rangle$, is transmitted to the leader. We suppose there are no communication delays.

\section{LEADER-FOLLOWER KINEMATIC MODEL}

The leader-follower setup consists of $q+1$ nonholonomic mobile robots whose individual kinematics can be abstracted as a unicycle model,

$$
\dot{x}=v \cos \theta, \quad \dot{y}=v \sin \theta, \quad \dot{\theta}=\omega
$$

where $(x, y)$ represents the position and $\theta$ the orientation of the robot with respect to the world frame $\langle W F\rangle$ (Fig. 2). One of these robots is the leader $\langle L\rangle$ whose configuration vector in the world frame is $\left[\begin{array}{lll}x_{L} & y_{L} & \theta_{L}\end{array}\right]^{T}$. The other $q$ robots are the followers, $\left\langle F_{i}\right\rangle$, described by $\left[\begin{array}{lll}x_{F_{i}} & y_{F_{i}} & \theta_{F_{i}}\end{array}\right]^{T}$, $i=1, \ldots, q$. The control inputs are the translational and angular velocities $\left[v_{L} \omega_{L}\right]^{T}$ and $\left[v_{F_{i}} \omega_{F_{i}}\right]^{T}$ of the leader and followers, respectively.

The view-angle $\psi_{i}$ is the angle from the leader $y$-axis to the line connecting the centers of mass of the leader and the $i$ th follower (the view-angle $\eta_{i}$ is defined in a similar fashion). In the spirit of [5] we assume that each robot is characterized by a different color and is equipped with an omnidirectional camera, whose coordinate system is supposed to be coincident with that of the robot. However, differently from [5], [16], we do not assume any knowledge of the camera calibration parameters (i.e. mirror shape, focal length, pixel aspect ratio, etc.). The robot centroid $C_{i}$ (Fig. 3(a)) can be detected in the image using standard color filtering techniques. After the centroid detection, the viewangle is readily computed as in Fig. 3(b).

The whole leader-follower setup can be modelled using polar coordinates [5], where $\rho_{i}$ is the distance from the center of the leader $\langle L\rangle$ and the $i$ th follower $\left\langle F_{i}\right\rangle$. Let us refer to $\beta_{i}$ as the relative orientation between the robots, then $\beta_{i}=-\psi_{i}+\eta_{i}+\pi$.

Proposition 1 (One leader - one follower kinematics): Consider the setup in Fig. 2. The one leader - one follower kinematics can be written as follows:

$$
\dot{\mathbf{s}}_{i}=\mathbf{G}_{i}\left(\mathbf{s}_{i}\right) \mathbf{u}_{i}, \quad i=1, \ldots, q
$$

where

$$
\mathbf{G}_{i}\left(\mathbf{s}_{i}\right)=\left[\begin{array}{cccc}
\cos \gamma_{i} & 0 & -\cos \psi_{i} & 0 \\
\frac{-\sin \gamma_{i}}{\rho_{i}} & 0 & \frac{\sin \psi_{i}}{\rho_{i}} & -1 \\
0 & -1 & 0 & 1
\end{array}\right]
$$
being $\quad \mathbf{u}_{i}=\left[\begin{array}{llll}v_{F_{i}} & \omega_{F_{i}} & v_{L} & \omega_{L}\end{array}\right]^{T}, \quad \mathbf{s}_{i}=\left[\begin{array}{lll}\rho_{i} & \psi_{i} & \beta_{i}\end{array}\right]^{T}$
and $\gamma_{i} \triangleq \beta_{i}+\psi_{i}$.

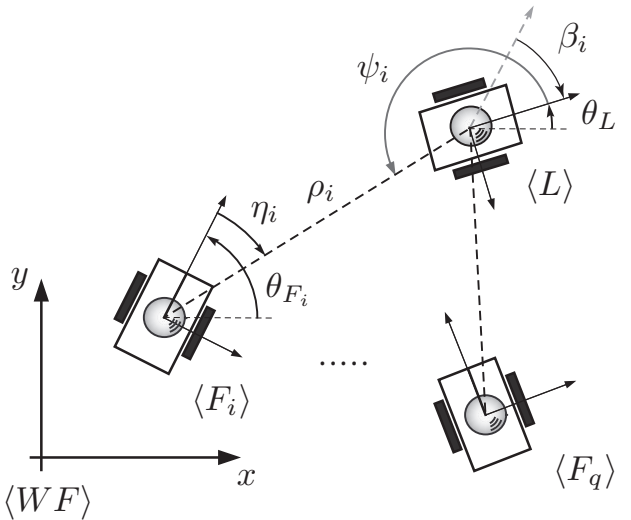

Fig. 2. Basic leader-follower setup in polar coordinate representation. Panoramic cameras provide information on robots relative orientation.

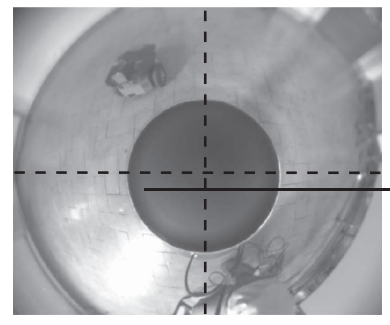

(a)

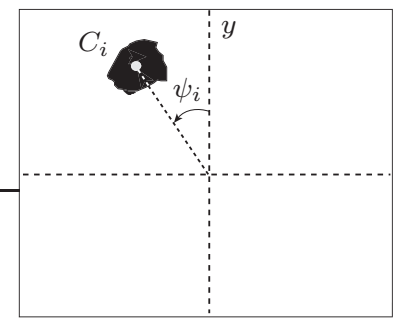

(b)
Fig. 3. Vision sensing. (a) Panoramic image provided by the leader; (b) Detection of the centroid $C_{i}$ and computation of the view-angle $\psi_{i}$. A coarse approximation of the principal point can be provided by the image center. 
The one leader - many follower kinematics is in [14] and can be obtained extending the one leader - one follower case in Prop. 1:

$$
\dot{\mathbf{s}}=\mathbf{G}(\mathbf{s}) \mathbf{u}
$$

where $\quad \mathbf{u}=\left[\begin{array}{lllllll}v_{F_{1}} & \omega_{F_{1}} \ldots & v_{F_{q}} & \omega_{F_{q}} & v_{L} & \omega_{L}\end{array}\right]^{T} \in \mathbb{R}^{2(q+1)}$ and $\mathbf{s}=\left[\begin{array}{lll}\mathbf{s}_{1}^{T} & \ldots & \mathbf{s}_{q}^{T}\end{array}\right]^{T} \in \mathbb{R}^{3 q}$.

\section{VISION-BASED OBSERVABILITY OF LEADER-FOLLOWER FORMATIONS}

\section{A. Basics on nonlinear observability}

We here present some basic facts about observability of nonlinear systems [11], [12], in order to study the localizability of leader-follower formations in the case of panoramic cameras used as the only sensor system. Prop. 2 provides an analytical sufficient condition for the observability in our multirobot context.

Consider a generic nonlinear system $\Sigma_{N}$ of the form

$$
\Sigma_{N}:\left\{\begin{array}{l}
\dot{\mathbf{s}}(t)=\mathbf{f}(\mathbf{s}(t), \mathbf{u}(t)), \mathbf{s}(0)=\mathbf{s}_{0} \in \mathbb{R}^{n} \\
\mathbf{y}(t) \triangleq \mathbf{h}(\mathbf{s}(t))=\left[\begin{array}{llll}
h_{1} & h_{2} \ldots h_{m}
\end{array}\right]^{T}
\end{array}\right.
$$

where $\mathbf{s}(t)=\left[s_{1}(t) s_{2}(t) \ldots s_{n}(t)\right]^{T} \in \mathcal{S}$ is the system state, $\mathbf{y}(t) \in \mathcal{Y}$ the observation vector and $\mathbf{u}(t) \in \mathcal{U}$ the input. $\mathcal{S}, \mathcal{Y}$ and $\mathcal{U}$ are differential manifolds of dimension $n, m$ and $p$, respectively.

Let $\lambda(\mathbf{s}): \mathbb{R}^{n} \mapsto \mathbb{R}$ be a scalar-valued function and define the following operator,

$$
d \lambda(\mathbf{s}) \triangleq \frac{\partial \lambda(\mathbf{s})}{\partial \mathbf{s}}=\left[\frac{\partial \lambda(\mathbf{s})}{\partial s_{1}} \frac{\partial \lambda(\mathbf{s})}{\partial s_{2}} \ldots \frac{\partial \lambda(\mathbf{s})}{\partial s_{n}}\right] .
$$

The problem of observability for $\Sigma_{N}$ can be roughly viewed as the injectivity of the input-output map $\mathcal{R}_{\Sigma_{N}}: \mathcal{S} \times \mathcal{U} \mapsto \mathcal{Y}$ with respect to the initial conditions. Two states $\mathbf{s}_{1}$ and $\mathbf{s}_{2}$ are said indistinguishable [15], $\mathbf{s}_{1} I \mathbf{s}_{2}$, if $\mathbf{y}_{\mathbf{s}_{1}, \mathbf{u}(t)}=\mathbf{y}_{\mathbf{s}_{2}, \mathbf{u}(t)}$, i.e., there exists an input $\mathbf{u}(t)$ and a time $t$ for which, starting from different $\mathbf{s}_{1}$ and $\mathbf{s}_{2}, \Sigma_{N}$ exhibits the same outputs.

The concepts of observability and indistinguishability are related, as shown in the following:

Definition 1 (Observability [15]): Given two states $\mathbf{s}_{1}, \mathbf{s}_{2} \in \mathcal{S}$, system $\Sigma_{N}$ is observable, if $\mathbf{s}_{1} I \mathbf{s}_{2} \Rightarrow \mathbf{s}_{1}=\mathbf{s}_{2}$.

An analytical sufficient condition for the local weak observability of $\Sigma_{N}$ has been introduced by Hermann and Krener in [9]. An equivalent and more intuitive formulation, based on the Extended Output Jacobian (EOJ), was proposed in [14] and here reported.

Proposition 2: System $\Sigma_{N}$ is said to be locally weakly observable at a point $\mathbf{s}^{o} \in \mathbb{R}^{n}$, if there exists an open set $\mathcal{D} \subset \mathbb{R}^{n}$ of $\mathbf{s}^{o}$ such that, for arbitrary $\mathbf{s} \in \mathcal{D}$, the set of row vectors,

$$
\left\{d h_{i}^{(j-1)}(\mathbf{s}) \mid i=1, \ldots, m ; j=1, \ldots, n\right\}
$$

is linearly independent ( $j$ is the order of time differentiation). From (3) we define the Extended Output Jacobian matrix $\mathbf{J} \in \mathbb{R}^{m n \times n}$, built by stacking the row vectors in (3).

Remark 1: Roughly speaking Prop. 2 states that the observability of $\Sigma_{N}$ can be tested by checking the rank of a matrix made of the state partial derivatives of the output vector and of all its $n-1$ time derivatives. From Prop. 2, it is straightforward to note that the system observability holds true also when at least one $n \times n$ submatrix of $\mathbf{J}$ has full rank. Thus, it is not necessary to check for the determinants of all possible submatrices of $\mathbf{J}$.

\section{B. Observability condition for leader-follower formations}

Proposition 2 is here used to provide an analytical condition for the observability of the setup presented in Sect. II in the case of a single follower. As previously discussed, we assume that the leader can observe the $i$ th follower and measure a two dimensional output vector,

$$
\mathbf{y}_{i} \triangleq\left[\begin{array}{ll}
y_{1} & y_{2}
\end{array}\right]^{T}=\left[\begin{array}{ll}
\psi_{i} & \beta_{i}
\end{array}\right]^{T} .
$$

From Prop. 2, the observability of (1) with output (4), is guaranteed when at least one $3 \times 3$ submatrix of the whole Extended Output Jacobian $\mathbf{J} \in \mathbb{R}^{6 \times 3}$ is nonsingular. Consider, e.g., the submatrix $\mathbf{J}_{i}$,

$$
\mathbf{J}_{i}=\left[\begin{array}{ccc}
\frac{\partial y_{1}}{\partial \rho_{i}} & \frac{\partial y_{1}}{\partial \psi_{i}} & \frac{\partial y_{1}}{\partial \beta_{i}} \\
\frac{\partial \dot{y}_{1}}{\partial \rho_{i}} & \frac{\partial \dot{y}_{1}}{\partial \psi_{i}} & \frac{\partial \dot{y}_{1}}{\partial \beta_{i}} \\
\frac{\partial y_{2}}{\partial \rho_{i}} & \frac{\partial y_{2}}{\partial \psi_{i}} & \frac{\partial y_{2}}{\partial \beta_{i}}
\end{array}\right]=\left[\begin{array}{ccc}
0 & 1 & 0 \\
\frac{\partial \dot{\psi}_{i}}{\partial \rho_{i}} & \frac{\partial \dot{\psi}_{i}}{\partial \psi_{i}} & \frac{\partial \dot{\psi}_{i}}{\partial \beta_{i}} \\
0 & 0 & 1
\end{array}\right]
$$

whose determinant is

$$
\operatorname{det}\left(\mathbf{J}_{i}\right)=-\frac{\partial \dot{\psi}_{i}}{\partial \rho_{i}}=\frac{1}{\rho_{i}}\left[\dot{\psi}_{i}+\omega_{L}\right] .
$$

Therefore, if $\operatorname{det}\left(\mathbf{J}_{i}\right) \neq 0$ the state $\mathbf{s}_{i}(t)$ is observable.

In the case of $q$ followers the observability condition is a simple extension of (5), as shown in [14].

1) A geometrical interpretation of the EOJ singularity: In Fig. 4 we provide a basic example to give a geometrical interpretation of (5). The leader $\langle L\rangle$ observes two moving followers $\left\langle F_{1}\right\rangle$ and $\left\langle F_{2}\right\rangle$ at different time instants $t=0$ and $t=1$. Intuitively, one can argue that any change in the visual data represents a gain in information and can thus improve the localization process. The following arguments, based on (5), confirm this intuition. At $t=0,\left\langle F_{1}\right\rangle$ has the same heading of $\langle L\rangle$ (i.e., $\beta_{1}(0)=0$ ), while $\left\langle F_{2}\right\rangle$ has not $\left(\beta_{2}(0) \neq 0\right)$. Suppose that $\psi_{1}(0)=\psi_{2}(0)$ and that all the robots move with the same translational velocity. At time $t=1$ it happens that $\psi_{1}(1)=\psi_{1}(0)$ (i.e., no temporal change in visual information) while $\psi_{2}(1) \neq \psi_{2}(0)$ and thus,

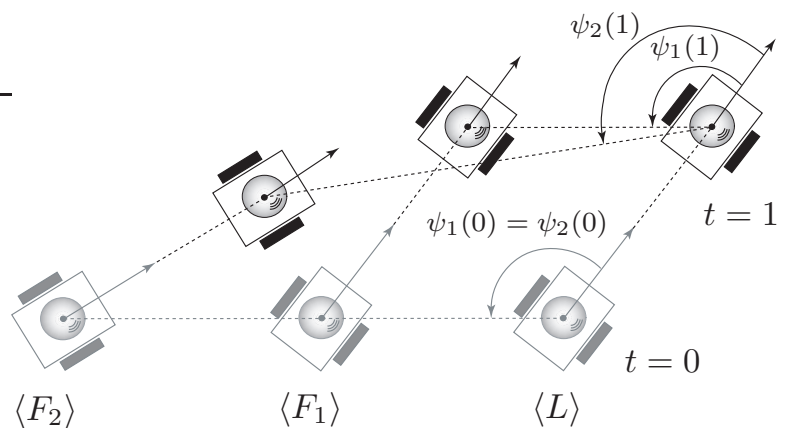

Fig. 4. Geometrical interpretation of the EOJ singularity. 
$\dot{\psi}_{1}=0$ and $\dot{\psi}_{2} \neq 0$. This implies, from (5), that $\operatorname{det}\left(\mathbf{J}_{2}\right) \neq 0$, while $\operatorname{det}\left(\mathbf{J}_{1}\right)=0$. As a conclusion, from Prop. 2, the state of $\left\langle F_{2}\right\rangle$ is observable, while we can argue that the state of $\left\langle F_{1}\right\rangle$ is not observable.

\section{NONLINEAR OBSERVER}

In order to control the formation with $q$ followers we need an estimate $\hat{\mathbf{s}}$ of the state configuration $\mathbf{s}$. This estimate is provided by a nonlinear observer that uses the input vector $\mathbf{u}$ and the output vector

$$
\mathbf{y} \triangleq\left[\begin{array}{lll}
\mathbf{y}_{1}^{T} & \ldots & \mathbf{y}_{q}^{T}
\end{array}\right]^{T} \in \mathbb{R}^{2 q} .
$$

We assume additive noise on both the process (2) and measurement equation (6),

$$
\begin{aligned}
\dot{\mathbf{s}} & =\mathbf{G}(\mathbf{s}) \mathbf{u}+\mathbf{z} & \text { with } & \mathbf{z} \sim \operatorname{WGN}(\mathbf{0}, \mathbf{Q}) \\
\mathbf{y} & =\mathbf{C s}+\mathbf{v} & \text { with } & \mathbf{v} \sim \operatorname{WGN}(\mathbf{0}, \mathbf{R})
\end{aligned}
$$

where $\mathbf{C}$ is the output transition matrix, $\mathbf{z}$ and $\mathbf{v}$ are white gaussian noises (WGN) with zero mean and covariance matrices $\mathbf{Q}$ and $\mathbf{R}$, respectively. We assume that $\mathbf{s}(0), \mathbf{z}$ and $\mathbf{v}$ are uncorrelated. We apply the Euler forward method with sampling time $T_{c}$ to discretize (7),

$$
\mathbf{s}(k+1)=\mathbf{\Gamma}(\mathbf{s}(k), \mathbf{u}(k))+T_{c} \mathbf{z}
$$

where $\boldsymbol{\Gamma}(\mathbf{s}(k), \mathbf{u}(k)) \triangleq T_{c} \mathbf{G}(\mathbf{s}) \mathbf{u}+\mathbf{s}(k)$ and $k \in \mathbb{N}$. In the next paragraph we describe the Unscented Kalman Filter (UKF), the nonlinear observer we used to estimate the state $\mathbf{s}$.

\section{A. Unscented Kalman Filter}

The UKF [13] represents a derivative-free alternative to the extended Kalman filter (EKF) in the framework of robot localization. Since Jacobian matrices need not to be computed, then the filter equations do not change as the system state dimension grows. This property is particularly suited in our multirobot framework, since the observer remains unchanged when new followers join the formation. The UKF has a computational complexity cubic in the size of the state vector, the same order of magnitude of the EKF. Nonetheless, the UKF handles system nonlinearities with higher accuracy than the EKF. Analogously to the Kalman filter, the UKF consists of a prediction and a correction step [17], that are detailed in the Appendix.

To the best of our knowledge, this is the first time that the $\mathrm{UKF}$ is applied to localize vision-based multirobot formations. The connections between the observability condition and the UKF performances will be discussed in Sect. VI.

\section{FEEDBACK CONTROL VIA DYNAMIC EXTENSION}

Consider the set of kinematic equations equivalent to (1),

$$
\begin{aligned}
\dot{\mathbf{s}}_{r_{i}} & =\mathbf{F}_{i}\left(\mathbf{s}_{i}\right) \mathbf{u}_{L}+\mathbf{H}_{i}\left(\mathbf{s}_{i}\right) \mathbf{u}_{F_{i}} \\
\dot{\beta}_{i} & =\omega_{L}-\omega_{F_{i}},
\end{aligned}
$$

where $\mathbf{s}_{r_{i}}=\left[\begin{array}{ll}\rho_{i} & \psi_{i}\end{array}\right]^{T}, i=1, \ldots, q$, is a reduced state-space vector and $\mathbf{H}_{i} \in \mathbb{R}^{2 \times 2}, \mathbf{F}_{i} \in \mathbb{R}^{2 \times 2}$ consist of the first and the last two columns of $\mathbf{G}_{i}$, respectively.
Using an analogous expression of (9), the authors in [5] proposed an input-state feedback linearizing control law assuming that a landmark was displaced at a known distance w.r.t. the robot centroid. In this way the invertibility of $\mathbf{H}_{i}$ is guaranteed and, as a consequence, also the feasibility of the control scheme. However, this assumption restricts the applicability of their formation control scheme only to near robots, for which the displaced landmark can be easily detected.

In order to overcome this problem, as anticipated in Sect. II, we propose here to observe the centroid of the robot. Despite the singularity of $\mathbf{H}_{i}$, it is yet possible to design a feedback control via dynamic extension [6], [12] with output vector $\left[\begin{array}{ll}\rho_{i} & \psi_{i}\end{array}\right]^{T}$ by adding integrators on a subset of the input channels. A time differentiation of the first two equations in (1), yields,

$$
\begin{aligned}
& {\left[\begin{array}{l}
\ddot{\rho}_{i} \\
\ddot{\psi}_{i}
\end{array}\right]=\left[\begin{array}{l}
-\dot{v}_{L} \cos \psi_{i}+v_{L} \dot{\psi}_{i} \sin \psi_{i} \\
\frac{\rho_{i}\left[\dot{v}_{L} \sin \psi_{i}+v_{L} \dot{\psi}_{i} \cos \psi_{i}\right]-v_{L} \dot{\rho}_{i} \sin \psi_{i}}{\rho_{i}^{2}}-\dot{\omega}_{L}
\end{array}\right]+} \\
& +\left[\begin{array}{l}
\dot{v}_{F_{i}} \cos \gamma_{i}-v_{F_{i}} \dot{\gamma}_{i} \sin \gamma_{i} \\
-\frac{\rho_{i}\left[\dot{v}_{F_{i}} \sin \gamma_{i}+v_{F_{i}} \dot{\gamma}_{i} \cos \gamma_{i}\right]-v_{F_{i}} \dot{\rho}_{i} \sin \gamma_{i}}{\rho_{i}^{2}}
\end{array}\right] .
\end{aligned}
$$

Substituting the first two lines of (1) in (11) and collecting the terms depending on the new input $\boldsymbol{\mu} \triangleq\left[\begin{array}{ll}\dot{v}_{F_{i}} & \omega_{F_{i}}\end{array}\right]^{T}$, we obtain,

$$
\begin{aligned}
& {\left[\begin{array}{c}
\ddot{\rho}_{i} \\
\ddot{\psi}_{i}
\end{array}\right]=\underbrace{\left[\begin{array}{cc}
\cos \gamma_{i} & v_{F_{i}} \sin \gamma_{i} \\
-\frac{\sin \gamma_{i}}{\rho_{i}} & \frac{v_{F_{i}} \cos \gamma_{i}}{\rho_{i}}
\end{array}\right]}_{\mathbf{C}\left(\mathbf{s}, v_{F_{i}}\right)}\left[\begin{array}{l}
\dot{v}_{F_{i}} \\
\omega_{F_{i}}
\end{array}\right]+} \\
& +\left[\begin{array}{l}
\frac{1}{\rho_{i}}\left[v_{L} \sin \psi_{i}-v_{F_{i}} \sin \gamma_{i}\right]^{2}-\dot{v}_{L} \cos \psi_{i}- \\
\frac{2}{\rho_{i}^{2}}\left[v_{F_{i}}^{2} \cos \gamma_{i} \sin \gamma_{i}+v_{L}^{2} \cos \psi_{i} \sin \psi_{i}\right]-\dot{\omega}_{L}-
\end{array}\right. \\
& -v_{L} \omega_{L} \sin \psi_{i} \\
& \left.-\frac{2 v_{L} v_{F_{i}} \sin \left(\gamma_{i}+\psi_{i}\right)}{\rho_{i}^{2}}+\frac{-v_{L} \omega_{L} \cos \psi_{i}+\dot{v}_{L} \sin \psi_{i}}{\rho_{i}}\right] .
\end{aligned}
$$

Supposing $v_{F_{i}} \neq 0$, then $\mathbf{C}\left(\mathbf{s}, v_{F_{i}}\right)$ represents a nonsingular counterpart of the decoupling matrix $\mathbf{H}_{i}\left(\mathbf{s}_{i}\right)$, being $\operatorname{det}\left(\mathbf{C}\left(\mathbf{s}, v_{F_{i}}\right)\right)=v_{F_{i}} / \rho_{i}$. Since $\mathbf{C}\left(\mathbf{s}, v_{F_{i}}\right)$ is invertible, the following output-tracking control law can be implemented,

$$
\boldsymbol{\mu}=\mathbf{C}\left(\mathbf{s}, v_{F_{i}}\right)^{-1}\left[\boldsymbol{\nu}-\mathbf{b}\left(\mathbf{s}, v_{F_{i}}\right)\right],
$$

where $\mathbf{b}\left(\mathbf{s}, v_{F_{i}}\right)$ is the second term on the right-hand side of (12) and $\nu$ is:

$$
\left[\begin{array}{l}
\nu_{1} \\
\nu_{2}
\end{array}\right] \triangleq\left[\begin{array}{l}
\ddot{\rho}_{i}^{\text {des }}+k_{i_{21}}\left(\dot{\rho}_{i}^{\text {des }}-\dot{\rho}_{i}\right)+k_{i_{11}}\left(\rho_{i}^{\text {des }}-\rho_{i}\right) \\
\ddot{\psi}_{i}^{\text {des }}+k_{i_{22}}\left(\dot{\psi}_{i}^{\text {des }}-\dot{\psi}_{i}\right)+k_{i_{12}}\left(\psi_{i}^{\text {des }}-\psi_{i}\right)
\end{array}\right]
$$

where $\rho_{i}^{\text {des }}$ and $\psi_{i}^{\text {des }}$ are the desired values. The polynomials $z^{2}+k_{i_{2 j}} z+k_{i_{1 j}}, j=1,2$, are Hurwitz and $k_{i_{1 j}}, k_{i_{2 j}} \in \mathbb{R}$ are the controller gains. In order to compute the actuating control input $v_{F_{i}}$, the signal $\dot{v}_{F_{i}}$ has to be integrated at each time $t$. Note the following points: 
i) In order to guarantee the closed loop stability the study of the internal dynamics is required. In particular, due to the dynamic extension process, the extended state-space dimension is $\tau=4$ and the system is described by,

$\left[\begin{array}{c}\dot{\rho}_{i} \\ \dot{\psi}_{i} \\ \dot{\beta}_{i} \\ \dot{v}_{F_{i}}\end{array}\right]=\left[\begin{array}{cc}0 & 0 \\ 0 & 0 \\ 0 & -1 \\ 1 & 0\end{array}\right]\left[\begin{array}{c}\dot{v}_{F_{i}} \\ \omega_{F_{i}}\end{array}\right]+\left[\begin{array}{c}v_{F_{i}} \cos \gamma_{i}-v_{L} \cos \psi_{i} \\ \frac{-v_{F_{i}} \sin \gamma_{i}+v_{L} \sin \psi_{i}}{\rho_{i}}-\omega_{L} \\ \omega_{L} \\ 0\end{array}\right]$.

However from (12), the total relative degree of (14) is 4 (for both the outputs $\rho_{i}, \psi_{i}$ ), that equals $\tau$. Then, we conclude that (14) has not internal dynamics and the output tracking control law (13) yields full-state linearization.

ii) In order to initialize the dynamic controller for an exact reproduction of the desired output trajectories, a common choice consists in assuming $\rho_{i}(0)=\rho_{i}^{\text {des }}(0)$ and $\psi_{i}(0)=\psi_{i}^{\text {des }}(0)$. Exploiting $\tan \gamma_{i}$ from the first two lines of (1), and the leader's known control inputs $v_{L}(0), \omega_{L}(0)$, we get,

$$
\begin{array}{r}
\beta_{i}(0)=\operatorname{ATAN} 2\left\{v_{L}(0) \sin \left(\psi_{i}(0)\right)-\rho_{i}(0)\left(\omega_{L}(0)+\dot{\psi}_{i}(0)\right),\right. \\
\left.v_{L}(0) \cos \left(\psi_{i}(0)\right)+\dot{\rho}_{i}(0)\right\}-\psi_{i}(0) .
\end{array}
$$

Analogously, we obtain,

$$
v_{F_{i}}(0)=\frac{\dot{\rho}_{i}(0)+v_{L}(0) \cos \left(\psi_{i}(0)\right)}{\cos \left(\beta_{i}(0)+\psi_{i}(0)\right)} .
$$

\section{Simulation RESUlts}

This section presents the simulation experiments we carried out to analyze the performance of the closed-loop system and to study condition (5). For the sake of simplicity the formation consists of two followers. We set $v_{L}(t)=5 \mathrm{~m} / \mathrm{s}$ and $\omega_{L}(t)=0 \mathrm{rad} / \mathrm{s}$ if $t \in[0,2] \cup[6,8] \mathrm{s}, \omega_{L}(t)=\pi / 4 \mathrm{rad} / \mathrm{s}$ otherwise. Note that the trajectory generated by $\left[\begin{array}{ll}v_{L} & \omega_{L}\end{array}\right]^{T}$ is particularly suited to test condition (5), (remind the geometrical interpretation in Sect. III-B.1). The initial configuration vectors are $\left[\begin{array}{lll}x_{L}(0) & y_{L}(0) & \theta_{L}(0)\end{array}\right]^{T}=\left[\begin{array}{lll}5 & 2 & \pi / 2\end{array}\right]^{T}$, $\left[\begin{array}{lll}x_{F_{1}}(0) & y_{F_{1}}(0) & \theta_{F_{1}}(0)\end{array}\right]^{T}=\left[\begin{array}{lll}4.7 & 1.8 & \pi / 2\end{array}\right]^{T}$, $\left[\begin{array}{lll}x_{F_{2}}(0) & y_{F_{2}}(0) & \theta_{F_{2}}(0)\end{array}\right]^{T}=\left[\begin{array}{lll}5.3 & 1.8 & \pi / 2\end{array}\right]^{T}$, hence $\mathbf{s}(0)=\left[\begin{array}{llllll}0.361 & 2.159 & 0 & 0.361 & 4.124 & 0\end{array}\right]^{T}$. The UKF has been initialized with $\hat{\mathbf{s}}(0 \mid-1)=\left[\begin{array}{llllll}\frac{3}{2} \rho_{1}(0) & \psi_{1}(0) & \beta_{1}(0) & \frac{3}{2} \rho_{2}(0) & \psi_{2}(0) & \beta_{2}(0)\end{array}\right]^{T}$ corresponding to a $50 \%$ perturbation of the unknown distances to the leader and $\mathbf{P}(0 \mid-1)=10^{-2} \operatorname{diag}(1,1.1,1.1,1,1.1,1.1) . \quad$ The tuning parameters of the UKF (see the Appendix) are $\alpha=0.1, \kappa=0, \delta=-1$ and the sampling time $T_{c}=10 \mathrm{~ms}$. We set $\mathbf{Q}=10^{-7} \operatorname{diag}(1, \varrho, \varrho, 1, \varrho, \varrho)$ and $\mathbf{R}=10^{-7} \varrho \mathbf{I}_{4}$ where $\mathbf{I}_{4}$ is the $4 \times 4$ identity matrix and $\varrho=3.0462 \mathrm{rad}^{2}$. The desired state is $\mathbf{s}^{\text {des }}=\left[\begin{array}{lllllll}0.5 & 3 \pi / 4 & 0 & 0.5 & 5 \pi / 4 & 0\end{array}\right]^{T}$ and the controller gains are $k_{i_{11}}=k_{i_{12}}=0.15$, $k_{i_{21}}=k_{i_{22}}=0, i=1,2$.

Fig. 5(a) shows the trajectory of the leader and the followers maintaining the desired formation (in order to have a temporal reference in the figure the robots are drawn each second). Fig. 5(b) shows $\operatorname{det}\left(\mathbf{J}_{1}\right)\left(\operatorname{det}\left(\mathbf{J}_{2}\right)\right.$ is neglected being similar to $\left.\operatorname{det}\left(\mathbf{J}_{1}\right)\right)$. Note that the determinant is approximately equal to zero in correspondence of the rectilinear tracts of the trajectory, i.e., in the intervals $[0,2] \mathrm{s},[6,8] \mathrm{s}$. On the other hand, in the curvilinear tracts the determinant is significantly different to zero. Fig. 5(c) shows the errors $\rho_{1}-\hat{\rho}_{1}, \rho_{2}-\hat{\rho}_{2}$ (solid) and the $99.7 \%$ confidence intervals $\pm 3 \sqrt{\mathbf{P}_{(j, j)}}, j=1,4$ (dash). $\beta_{i}-\hat{\beta}_{i}$ and $\psi_{i}-\hat{\psi}_{i}, i=1,2$ are not given since angles (differently from distances) are not critical parameters to be estimated. From Fig. 5(c) we observe that in correspondence of the rectilinear tracts of the trajectory, the confidence intervals increase sensibly and the estimation errors are not equal to zero. Namely, the UKF localization gets worse (consistency is temporarily lost). This allows us to postulate that, besides a sufficient condition, Prop. 2 also provides a necessary condition for nonlinear observability, namely, if the EOJ is singular then the system is not observable. Although the previous results are sound, further investigations are needed to validate this hypothesis.

\section{CONCLUSIONS AND FUTURE WORKS}

In this paper we address both the localization and control problems for a centralized leader-follower formation of mobile robots equipped with panoramic vision sensors. Extending earlier works, we assume that the cameras are uncalibrated and that only the robots centroid needs to be observed in order to maintain the formation. The localizability problem is approached using a sufficient condition based on the Extended Output Jacobian matrix, while the Unscented Kalman Filter achieves the localization. A feedback control based on dynamic extension is designed in order to guarantee the leader-to-follower formation stability. Simulation experiments show the effectiveness of our coordination scheme also providing a meaningful insight into the observability condition. In particular, we postulated a stronger relationship between system observability and UKF performances.

Future research lines include the study of a decentralized control strategy and the extension of our results to formations with more involved robot kinematic models.

\section{APPENDIX \\ UNSCENTED KALMAN FILTER}

The UKF is based on the Unscented Transformation (UT) [17], a method for computing the statistics of a random variable $\mathbf{s}$ (with mean $\overline{\mathbf{s}}$ and covariance $\mathbf{P}$ ) that undergoes a nonlinear transformation. A set of points and weights $\left\{\boldsymbol{S}_{j},\left[W_{j}^{(m)}, W_{j}^{(c)}\right]\right\}_{j=0}^{6 q}$, called sigma points, are chosen in a deterministic way so that their sample mean and covariance are respectively $\overline{\mathbf{s}}$ and $\mathbf{P}$. For $\xi=1, \ldots, 3 q$, according to [17],

$$
\begin{array}{ll}
\boldsymbol{S}_{0}=\overline{\mathbf{s}} & W_{0}^{(m)}=\frac{\lambda}{3 q+\lambda} \\
\boldsymbol{S}_{\xi}=\overline{\mathbf{s}}+(\sqrt{(3 q+\lambda) \mathbf{P}})_{\xi} & W_{0}^{(c)}=\frac{\lambda}{3 q+\lambda}+1+\delta-\alpha^{2} \\
\boldsymbol{S}_{\xi+3 q}=\overline{\mathbf{s}}-(\sqrt{(3 q+\lambda) \mathbf{P}})_{\xi} & W_{\{1 . .6 q\}}^{(m)}=W_{\{1 \ldots 6 q\}}^{(c)}=\frac{1}{2(3 q+\lambda)}
\end{array}
$$

where $\lambda=\alpha^{2}(3 q+\kappa)-3 q$ is a scaling parameter, $\alpha \in\left[10^{-4}, 1\right]$ determines the spread of the sigma points 


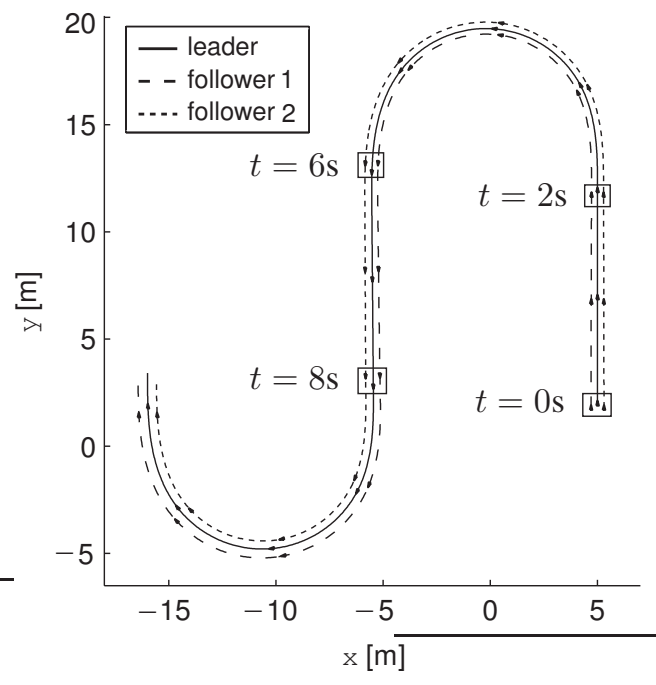

(a)

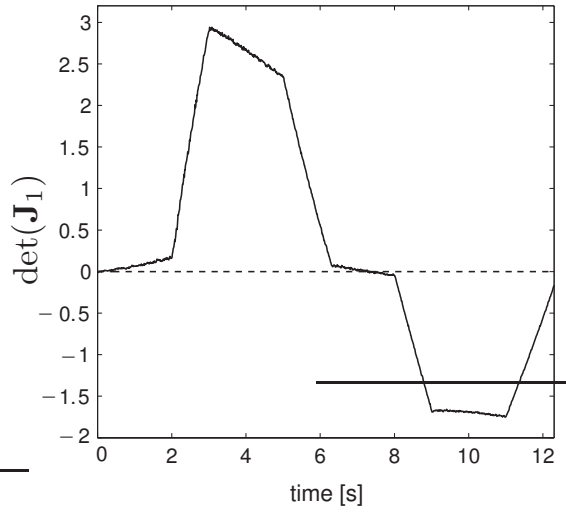

(b)
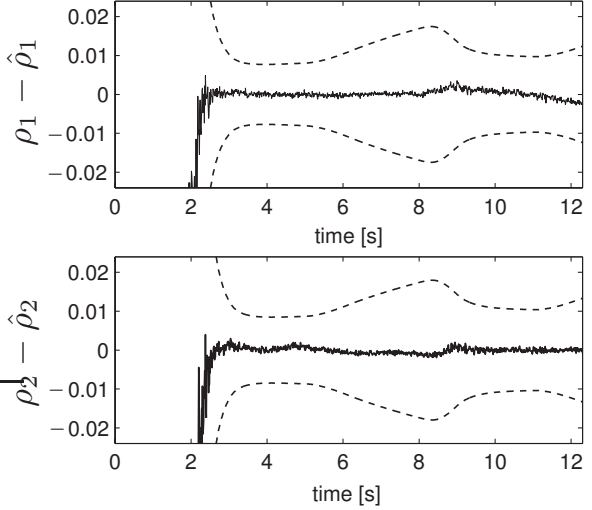

(c)

Fig. 5. (a) Trajectory of the robots; (b) Determinant of $\mathbf{J}_{1}$; (c) $\rho_{1}-\hat{\rho}_{1}, \rho_{2}-\hat{\rho}_{2}$ (solid) and $99.7 \%$ confidence intervals (dash).

around $\overline{\mathbf{s}}$ and $\kappa$ is a secondary scaling parameter which is usually set to 0 or $3-3 q . \delta$ is used to incorporate prior knowledge of the distribution of $\mathbf{s}(\delta=2$ is optimal for Gaussian distributions). $(\sqrt{(3 q+\lambda) \mathbf{P}})_{\xi}$ is the $\xi$ th column of the matrix $\sqrt{(3 q+\lambda) \mathbf{P}}$ (if a Cholesky factorization is used [13]). The UKF represents a straightforward extension of the UT in a recursive framework.

\section{Prediction Step}

1) Fix $\left\{W_{j}^{(m)}, W_{j}^{(c)}\right\}_{j=0}^{6 q}$ and compute $\boldsymbol{S}_{j}(k \mid k)$ in (15) using $\{\hat{\mathbf{s}}(k \mid k), \mathbf{P}(k \mid k)\}$. Transform $\boldsymbol{S}_{j}(k \mid k)$ according to the process model, thus obtaining $\boldsymbol{S}_{j}^{*}(k+1 \mid k)=$ $\boldsymbol{\Gamma}\left[\boldsymbol{S}_{j}(k \mid k), \mathbf{u}(k)\right]$.

2) The predicted mean and covariance are computed as, $\hat{\mathbf{s}}(k+1 \mid k)=\sum_{j=0}^{6 q} W_{j}^{(m)} \boldsymbol{S}_{j}^{*}(k+1 \mid k), \mathbf{P}(k+1 \mid k)=$ $\sum_{j=0}^{6 q} W_{j}^{(c)}\left\{\boldsymbol{S}_{j}^{*}(k+1 \mid k)-\hat{\mathbf{s}}(k+1 \mid k)\right\}\left\{\boldsymbol{S}_{j}^{*}(k+1 \mid k)\right.$ $-\hat{\mathbf{s}}(k+1 \mid k)\}^{T}+\mathbf{Q}$.

3) Compute $\boldsymbol{S}_{j}(k+1 \mid k)$ in (15) using $\{\hat{\mathbf{s}}(k+1 \mid k), \mathbf{P}(k+1 \mid k)\}$ and define the predicted points as, $\boldsymbol{Y}_{j}(k+1 \mid k)=\mathbf{C} \boldsymbol{S}_{j}(k+1 \mid k)$.

4) The predicted observation is $\hat{\mathbf{y}}(k+1 \mid k)=$ $\sum_{j=0}^{6 q} W_{j}^{(m)} \boldsymbol{Y}_{j}(k+1 \mid k)$.

5) The innovation covariance and the cross correlation matrices are $\mathbf{P}_{\mathbf{y y}}(k+1 \mid k)=$ $\sum_{j=0}^{6 q} W_{j}^{(c)}\left\{\boldsymbol{Y}_{j}(k+1 \mid k)-\hat{\mathbf{y}}(k+1 \mid k)\right\}\left\{\boldsymbol{Y}_{j}(k+1 \mid k)\right.$ $-\hat{\mathbf{y}}(k+1 \mid k)\}^{T}+\mathbf{R}$ and $\mathbf{P}_{\mathbf{s y}}(k+1 \mid k)=$ $\sum_{j=0}^{6 q} W_{j}^{(c)}\left\{\boldsymbol{S}_{j}(k+1 \mid k)-\hat{\mathbf{s}}(k+1 \mid k)\right\}\left\{\boldsymbol{Y}_{j}(k+1 \mid k)\right.$ $-\hat{\mathbf{y}}(k+1 \mid k)\}^{T}$.

\section{Correction Step}

$\hat{\mathbf{s}}(k+1 \mid k+1)=\hat{\mathbf{s}}(k+1 \mid k)+\mathbf{K}(k+1)[\mathbf{y}(k+1)-\hat{\mathbf{y}}(k+1 \mid k)]$

$\mathbf{P}(k+1 \mid k+1)=\mathbf{P}(k+1 \mid k)-\mathbf{K}(k+1) \mathbf{P}_{\mathbf{y y}}(k+1 \mid k) \mathbf{K}(k+1)^{T}$

where $\mathbf{K}(k+1)=\mathbf{P}_{\mathbf{s y}}(k+1) \mathbf{P}_{\mathbf{y y}}^{-1}(k+1)$.

\section{REFERENCES}

[1] A. Bicchi, D. Prattichizzo, A. Marigo, and A. Balestrino. On the observability of mobile vehicles localization. In IEEE Mediterrean Conference on Control and Systems, volume 1, 1998.

[2] F. Bullo and A. D. Lewis. Geometric Control of Mechanical Systems, volume 49 of Texts in Applied Mathematics. Springer, 2005.

[3] K.K. Chintalapudi, A. Dhariwal, R. Govindan, and G.S. Sukhatme. Ad-Hoc Localization Using Ranging and Sectoring. In IEEE INFOCOM, volume 4, pages 2662-2672, 2004.

[4] F. Conticelli, A. Bicchi, and A. Balestrino. Observability and nonlinear observers for mobile robot localization. In IFAC International Symposium on Robot Control, 2000.

[5] A.K. Das, R. Fierro, V. Kumar, J.P. Ostrowsky, J. Spletzer, and C. Taylor. A Vision-Based Formation Control Framework. IEEE Transactions on Robotics and Automation, 18(5):813-825, 2002.

[6] J. Descusse and C.H. Moog. Decoupling with dynamic compensation for strong invertible affine systems. International Journal of Control, 42(6):1387-1398, 1985.

[7] D.A. Forsyth and J. Ponce. Computer Vision: A Modern Approach. Prentice Hall, 2002.

[8] R. Grabowski and P. Khosla. Localization Techniques for a Team of Small Robots. In IEEE Conference on Intelligent Robots and Systems, volume 2, pages 1067-1072, 2001.

[9] R. Hermann and A. Krener. Nonlinear controllability and observability. IEEE Transactions on Automatic Control, AC-22(5):728-740, 1977.

[10] J.P. Hespanha. State estimation and control for systems with perspective outputs. In 41st IEEE Conference on Decision and Control, volume 2, pages 2208-2213, 2002.

[11] H. Inaba, A. Yoshida, R. Abdursul, and B.K. Ghosh. Observability of Perspective Dynamical Systems. In 39th IEEE Conference on Decision and Control, volume 5, pages 5157-5162, 2000.

[12] A. Isidori. Nonlinear Control Systems. Springer, 3rd edition, 1995.

[13] S.J. Julier and J.K. Uhlmann. Unscented filtering and nonlinear estimation. Proceedings of the IEEE, 92(3):401-422, 2004.

[14] G.L. Mariottini, G.J. Pappas, D. Prattichizzo, and K. Daniilidis. Vision-based Localization of Leader-Follower Formations. In 43rd IEEE Conference on Decision and Control, pages 635-640, 2005.

[15] H. Nijmeijer and A.J. van der Shaft. Nonlinear dynamical control systems. Springer, 1990.

[16] R. Vidal, O. Shakernia, and S. Sastry. Following the flock: Distributed formation control with omnidirectional vision-based motion segmentation and visual servoing. IEEE Robotics and Automation Magazine, 11(4):14-20, 2004.

[17] E.A. Wan and R. van der Merve. The Unscented Kalman Filter. In Simon Haykin, editor, Kalman Filtering and Neural Networks, Adaptive and Learning Systems for Signal Processing, Communications, and Control. John Wiley \& Sons, 2001. 